\title{
Alcohol and the developing adolescent brain: evidence review
}

\author{
A Carson \\ Consultant Neuropsychiatrist, Department of Medical Rehabilitation and Department of Clinical Neurosciences, NHS Lothian, and part-time \\ Senior Lecturer, Division of Psychiatry and Centre for Clinical Brain Studies, University of Edinburgh, Edinburgh, UK
}

KEYWORDS alchohol, addiction, cognitive neuroscience

DECLARATION OF INTERESTS AC is not a member of Scottish Health Action on Alcohol Problems (SHAAP) but was pleased to provide this summary on an unpaid basis. The article was discussed with SHAAP prior to publication but they had no control over content

\author{
Correspondence to A Carson \\ Robert Fergusson Unit \\ Royal Edinburgh Hospital \\ Morningside Terrace \\ Edinburgh EHIO 5HF \\ UK
}

e-mail alan.carson@nhslothian.scot. nhs.uk

Scotland has a difficult relationship with alcohol. When introducing the Scottish Government's Alcohol (Minimum Pricing) Bill, the then health minister Nicola Sturgeon, said: 'For too long, too many Scots have been drinking themselves into an early grave...It is no coincidence that as the affordability of alcohol has plummeted in recent decades, alcohol-related deaths, disease, crime and disorder have spiralled'.' Initially she suggested a level of 45p per unit struck the right balance between affordability and health benefits, and asked some politicians to reconsider their hostility to the Bill 'for the sake of our nation's health'. The Bill was finally passed, at 50p per unit, with broad cross-party support except for Labour abstentions.

Nicola Sturgeon undoubtedly deserves praise for speaking out when so many politicians should be ashamed of their silence, or their complicit support of such policies as supermarket discounting of alcohol, for fear of offending voters. Running one of the national brain injury units, I see the effects of this 'difficult relationship' every day. Among my current 19 inpatients, all with severe acquired brain injury, in approximately two-thirds of cases alcohol or drug misuse has played a significant aetiological role. These patients, typically young men, now have severe acquired cognitive impairment, difficulty speaking, swallowing and walking: a future of 30 years or more in care home awaits them an undoubted tragedy.

Professor Neil Douglas, a previous president of the Royal College of Physicians of Edinburgh, claimed alcohol misuse in Scotland had reached crisis point. 'Excessive drinking has increasingly become the norm. It is now our national shame'. ${ }^{2}$ It is not just the amount but also the nature of this drinking and who does it that seems to be changing. There is a strong anecdotal sense that teenage alcohol problems, and the resultant physical complications, are becoming more commonplace. Dr John Wilson, Vice-President of the Royal College of
Physicians of Edinburgh, summed up a general concern: 'The medical profession now sees serious alcohol related illnesses occurring at younger ages and there is a worrying trend of harm to women in their thirties none of this was evident 25-30 years ago. ${ }^{3}$

As the father of two teenage children, it is a concern I share clinically and socially. We routinely, probably hypocritically, end up discussing concerns over teenage drinking with fellow parents over a glass of claret; reflecting perhaps the worrying, and much more silent, increase among middle-age and middle class drinkers. Tales of teenage daughters returning from parties unable to stand up, and certainly unable to take care of themselves, are commonplace. Our dissonant attitudes reflect the complexities of alcohol and adolescents. Few parents wonder when to let their children take a couple of small 'baggies' of heroin to a party, 'but remember don't inject, darling'. But with alcohol the general feeling is one of a concern regarding the dangers of excess, jarring against a view of societal inclusion and expectations that as adults they will drink to some extent. To me it is not just about policy but about education.

And for education we need information. What do we currently know? On 27th September 2013, Scottish Health Action for Alcohol hosted a seminar where policymakers and experts from the fields of genetics, neuroscience and social psychology came together to review and discuss new and emerging medical and scientific research about the impact of alcohol on the adolescent brain and implications for policy and practice. ${ }^{3}$ I have summarised the key points below.

Adolescent brains really are different - what every parent suspected is now a scientific 'fact'. It was previously believed that the human brain was structurally mature in early childhood, but developments in neuroimaging have allowed studies showing the brain 
matures, both structurally and functionally, into the early twenties. In infancy the number of synaptic connections between neurones increases dramatically, but this is followed by a prolonged period of 'pruning' in which redundant connections and networks are discarded. This process of pruning seems to reflect the normal development of the child. During the prepubertal years it involves sensori-motor networks and vision, but in adolescence the major changes are in the tracts involved in executive function (planning, judgement, impulsivity) and social cognition.

These structural changes are mirrored in functional changes. Adolescents 'see' the world differently, as their brains respond with altered patterns to social cues. They seem particularly sensitive, and have more marked emotional responses, compared to adult brains, to scenarios that involve ostracism. Adults by contrast show better abilities to consider the long-term consequences of behaviour. The cognitive differences reflect underlying structural changes; the limbic system, which controls basic emotions and rewards based learning, matures faster than the executive networks for planning and self regulation. Both may be heavily influenced by nascent networks for social cognition; with adolescents' brains being particularly susceptible to the effects of the desire to avoid ostracism from friends. Experimental paradigms using driving simulation show that, left on their own, teenage boys are actually rather cautious drivers - it is when their friends are present that the caution goes out the window. The message to parents seems to be, you can let your son drive your car, just don't allow him to give a lift to any friends.

Understanding the importance of social cognition, and how adolescents respond, is likely to be key to any policy intervention. Interventions that push long-term consequences are unlikely to resonate with brains that are not wired up to think like that yet. Interventions that emphasise immediate social benefits, which are peer driven, may be much more effective. Thus as a parent I may feel I am 'doing my bit' cautiously introducing a glass of champagne at my children's first grown up 'Hogmanay' party, but actually it was probably meaningless. They were only interested in hearing 'insider' tales from a film director who was also there and considering the social currency these titbits would carry back at school; what will really matter is the social currency of alcohol with these same peers.

Adolescent brains also respond differently to alcohol. Human studies suggest that they are more susceptible to alcohol induced memory impairment but, at least if animal data are to be believed, less susceptible to the motor effects; a scenario which leaves adolescents less likely to experience the early warning signs of intoxication such as clumsiness and unsteadiness.
In cross-sectional studies, adolescent drinkers have reductions in volume of the hippocampus (the central area for spatial navigation and short and long term memory) and prefrontal cortex (the area for planning, logical reasoning, impulse control and social cognition). They also have reduced integrity of white matter tracts throughout the brain. One cannot be certain from crosssectional designs whether these changes are the demonstration of pre-existing neural organisations that pre-dispose to drinking behaviour or the results of a neurotoxic effect of alcohol. Animal studies suggest the latter but we need longitudinal imaging data to confirm this in humans. In the one study to date, alcohol use in adolescence was associated with reduced integrity of the superior longitudinal fasciculus, a tract regulating motor behaviour. In summary: while there are conflicting results from imaging studies the trend is to suggest that heavy alcohol use in adolescence is associated with nontrivial brain changes.

Over the last two decades there has been increasing recognition of the role of genetic susceptibility in the risk of developing alcohol problems, accounting for $50-60 \%$ of the risk. ${ }^{4}$ How this increased risk is actually mediated is a major question. Understanding the interplay of genetics with the environment and with impulsivity the interplay between inhibitory control and reward anticipation - is a hot topic. The ongoing IMAGEN study of 2000 adolescents in 17 centres across Europe aims to tackle these questions. Early results suggest young people with potentially problematic substance misuse are predisposed to risk taking. They also appear to have differing dopaminergic responses to a variety of psychoactive substances - dopamine is a neurotransmitter known to be intimately connected with reward and pleasure seeking. Hypofunction of a specific orbitofrontal cortical network was associated with likelihood of initiating drug use in early adolescence, and right inferior frontal activity was related to the speed of the inhibition process and use of illegal substances; activity in this network was associated with genetic variation in a norepinephrine transporter gene..$^{5}$

Clinically, the acute intoxication effects of alcohol are believed to be mediated via the GABA system, the brain's main inhibitory neurotransmitter. Alcohol increases GABA activity leading to reduced anxiety, slurred speech, sedation, disinhibition and, ultimately, reduced consciousness. Simultaneously, alcohol blocks the excitatory glutamatergic system. The brain responds to chronic consumption with up-regulation. Such hyperactivity is neurotoxic and intimately involved in withdrawal phenomena. But glutamate also plays an important role in shaping neuroplasticity and the major concern in adolescence is that chronic consumption leads to long term alterations to brain structure owing to the effects in a particularly vulnerable period. 
One hope is that an improved understanding of the neural circuits mediating alcohol's effects will lead to a new generation of pharmacological treatments; particularly to reduce the craving for alcohol that is the true hallmark of addiction. Some drugs have come to market but only an enthusiast could claim overwhelming success to date. However, with a more sophisticated understanding of the mechanisms underlying addiction, the prospect of at least adjunctive treatments may improve. Greater understanding of the neural mechanisms may also open the potential for an improvement in psychological approaches to treatment. Targeted forms of cognitive rehabilitation could be employed to moderate the harmful effects of cognitive consequences of alcohol on brain development and encourage normal brain maturation. While undoubtedly desirable, I suspect this approach is more likely to feature highly in grant applications and experimental paradigms, rather than make a substantive contribution to clinical practice in the near future.

There is clearly cause to be worried about the effects of alcohol on developing brains, but how great is the problem? Exposure to alcohol is commonplace; the surprise is perhaps that by age 15 almost a quarter of Scottish youngsters have never consumed any alcohol. What is much more of a concern is that around a third appear to drink regularly and consume significant amounts with a median of II units per week for I5-yearolds and 8 units per week for 13-years-olds. Heavy consumption and a younger age of consumption are linked with deprivation, with the effect being strongest in girls. There is a recent suggestion that there is now a slight year on year decrease in consumption. We may have seen a peak in consumption but this is not a reason for complacency, as paradoxically problems seem to be worsening among a subgroup of problem teenage drinkers. Alcohol still has a role in 15\% of deaths in adolescents and young adults, is involved in two-thirds of violent crime in the same age group and has strong associations with unplanned pregnancy and sexually transmitted diseases - and that is far, far too much.

There is however a fundamental problem. When I was young, getting drunk was fun and I am sure it still is. All the people I knew survived the experience and that is still the case. It seems to me that it will be difficult to prevent teenage drinking and I am not sure it is desirable to do so. Some seem to advocate a policy of replacement with other drugs, talking of an 'honest' debate about harm, but it seems to me that this just crystallises different prejudices - and one notes that those same 'honest' advocates skate over inconvenient findings like the schizophrenogenic problems of cannabis.

What should we do collectively? Societal controls such as minimum pricing and more rigorous age verification are important but will never be adequate on their own. We need to improve socio-cultural education but be aware of how to optimally deliver this. Newer and better treatments may come along but in the interim we need to enhance the quality and accessibility of the services that currently exist. We also need to think of the design of bars, clubs and restaurants - how and where we interact as a country with alcohol. I think how we talk about alcohol and portray it in the media is central. We are aware, looking at the examples of race, sexuality and more recently cigarettes, that we can deliberately change culture for the better.

When I was young, racist jokes were commonplace but now would rightly be condemned and potentially lead to a broadcaster losing their job. Yet we continue, via the medium of broadcasting, to legitimise drunkenness. After a sporting victory the broadcaster commonly asks the interviewee the next day 'how is your head?' Even with the great casualties of alcohol misuse such as George Best, a player many think was the greatest natural talent ever who gave up on his playing career at the early age of 27 largely due to his alcoholism, few people seem to be able to resist recounting and glorifying the tales of his drinking. Some regard trying to shift cultural attitudes in derogatory terms talking of 'political correctness gone mad' but I am all for a shift that has left it unacceptable to laugh at racial insults, and I will be equally all for it if it reduces the rate of serious injury and brain damage in future generations.

\section{REFERENCES}

I Nicola Sturgeon proposes $45 \mathrm{p}$ minimum alcohol price. The Telegraph 2 September 2010. http://www.telegraph.co.uk/news/ uknews/scotland/7977352/Nicola-Sturgeon-proposes-45pminimum-alcohol-price.html (accessed 6/2/20I5).

2 Alcohol abuse 'becoming epidemic'. BBC News 2 November 2006 http://news.bbc.co.uk/I/hi/scotland/6107844.stm (accessed 6/2/2015).

3 SHAAP. Alcohol and the Developing Adolescent Brain: Evidence Review. Edinburgh: SHAAP; 2014. http://www.shaap.org.uk/images/shaap_ developing_adolescents_brain_press.pdf

4 Dick DM, Bierut LJ. The genetics of alcohol dependence. Curr Psychiatry Rep 2006; 8: 15I-7. http://dx.doi.org/|0.1007/s| | 920 006-0015-1

5 5. Whelan R, Conrod PJ, Poline JB et al. Adolescent impulsivity phenotypes characterized by distinct brain networks. Nat Neurosci 2012; 15: 920-5. http://dx.doi.org//0.1038/nn.3092 